\title{
The Gas Attenuator of FLASH at DESY
}

\author{
Ulrich Hahn and Kai Tiedtke
}

Deutsches Elektronen-Synchrotron DESY, Notkestrasse 85, 22603 Hamburg, Germany

\begin{abstract}
FLASH (Free electron LASer at Hamburg) as a part of the Deutsches Elektronen Synchroton DESY is the first Free Electron Laser (FEL) user facility for VUV and soft X-ray coherent light experiments. The SASE (Self Amplification by Stimulated Emission) process generates ultra short coherent radiation pulses on the femtosecond time scale with peak powers in the GW range. Several experiments need reliable means to reduce the FEL intensity over many orders of magnitude without changing the photon beam characteristics. Since a reduction of the FEL intensity by variation of machine parameters is not appropriate, a windowless gas-filled cell in combination with differential pumping units is used for attenuating the FEL radiation. This attenuator is placed in the beamline in outside the experimental hall. The total length of the gas cell is $15 \mathrm{~m}$ and the maximum gas pressure, which can be handled by the differential pumping units, is about 0.1 mbar. The attenuation range of Nitrogen covers at least 5 orders of magnitude in the spectral range of 19 to $120 \mathrm{~nm}$ due to its large absorption cross section. Between 19 and $9 \mathrm{~nm}$ and for shorter wavelengths Xenon and Krypton can be used, respectively.
\end{abstract}

Keywords: Free electron laser, gas attenuator, soft X-ray, differential pumping

PACS: $41.60 . \mathrm{Cr}, 07.85 . \mathrm{Qe}$, 42.79.Ci

\section{INTRODUCTION}

Since 2005 the first FEL user facility for VUV and soft X-ray coherent light experiments FLASH is in operation at DESY [1]. The facility consists of a superconducting accelerator in combination with a $30 \mathrm{~m}$ long undulator producing highly intense photon pulses in the GW range. The experimental hall of the user facility is located approximately $30 \mathrm{~m}$ behind the last dipole magnet which separates the electron and the photon beam. The photon beam transport system delivers the FEL radiation under ultra high vacuum conditions to the five different end stations, which can be used alternatively.

At FLASH a gas attenuator system based on single photon absorption in a gas is in routine operation to attenuate

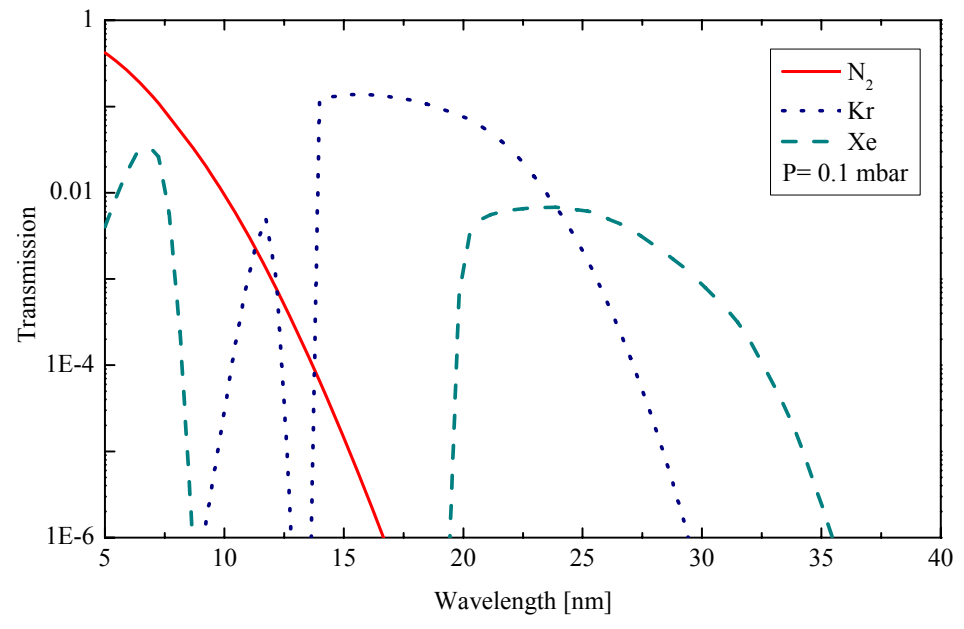

FIGURE 1. Calculated transmission of a $15 \mathrm{~m}$ long gas cell at a pressure of $0.1 \mathrm{mbar}$ for different gases.. the photon pulse energy (in average $10 \mu \mathrm{J}$ ) on user demand. Common examples are the alignment of samples at moderate pulse energies and the stepwise reduction of photon intensities without changing the focusing geometry in experiments investigating strong field phenomena. The principle of attenuation by photon absorption in a dilute gas combines several advantages such as:

i) Reduction of pulse energy without altering the photon beam characteristics

ii) Fast and flexible variation of the attenuation due to simple pumping schemes.

The attenuator consists of a windowless $15 \mathrm{~m}$ long gas cell in combination with two differential pumping units at both ends. The maximum gas pressure is $\sim 0.1$ mbar resulting in an attenuation range of at least 5 orders of

CP879, Synchrotron Radiation Instrumentation: Ninth International Conference, edited by Jae-Y oung Choi and Seungyu Rah

(c) 2007 A merican Institute of Physics 978-0-7354-0373-4/07/\$23.00 
magnitude. Figure 1 shows the calculated transmission of the gas cell at a pressure of $0.1 \mathrm{mbar}$ for $\mathrm{N}_{2}, \mathrm{Kr}$, and Xe.

In this paper, we report on the technical layout of the attenuator. In particular, the optimized design of the differential pumping units providing the required pressure gradient is described. First experimental results with a Nitrogen gas filling are presented.

\section{BASIC LAYOUT OF THE GAS ATTENUATOR}

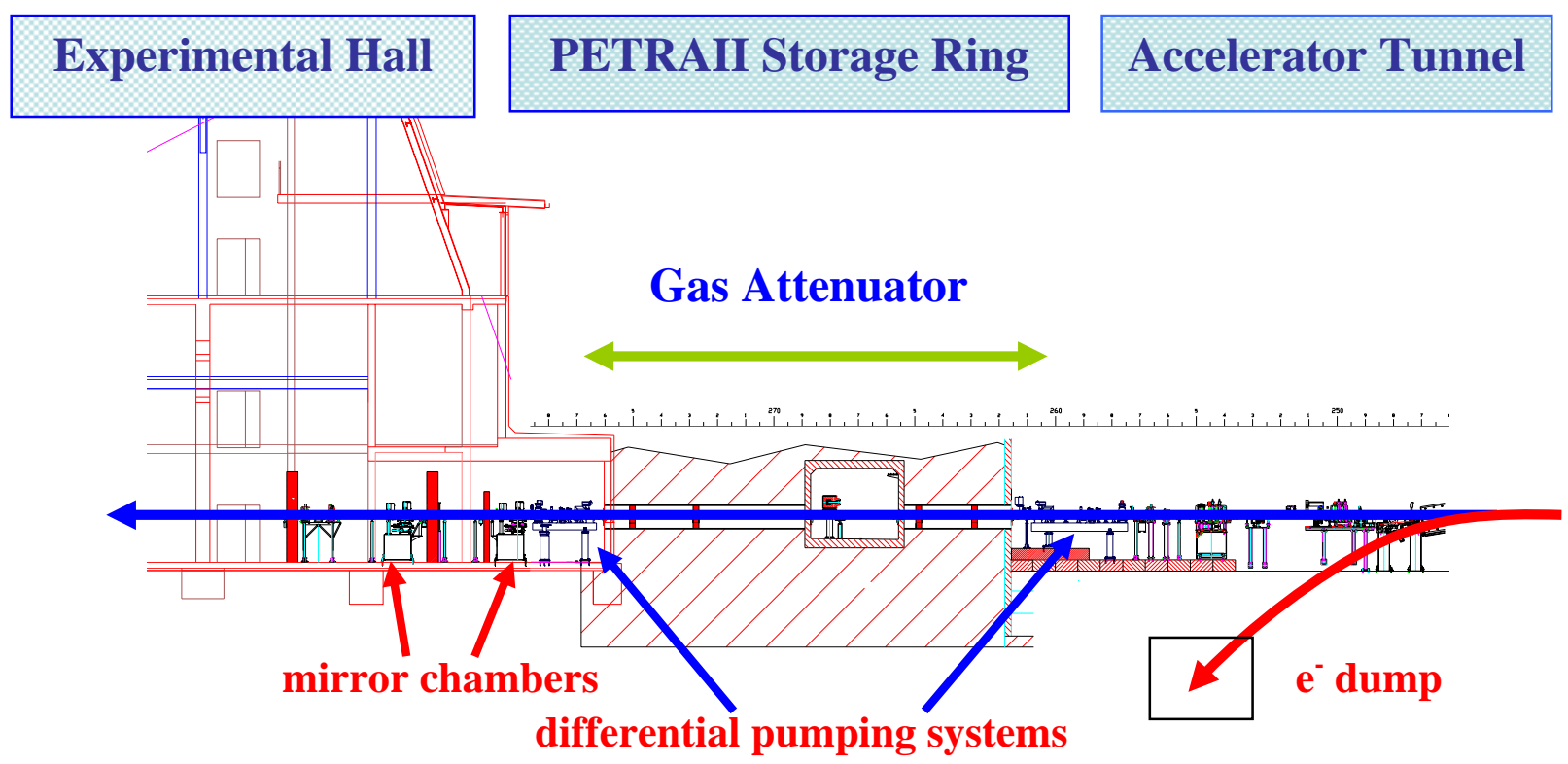

FIGURE 2. The layout of the gas attenuator at the crossing of the storage ring PETRA II and FLASH

Figure 2 shows the layout of the FLASH gas attenuator. On the right, the electron and photon beams are separated with the help of a dipole magnet. The FEL photons leave the accelerator tunnel and pass the storage ring PETRAII to attain the experimental hall. The $15 \mathrm{~m}$ long drift section through PETRAII is an ideal place to install a

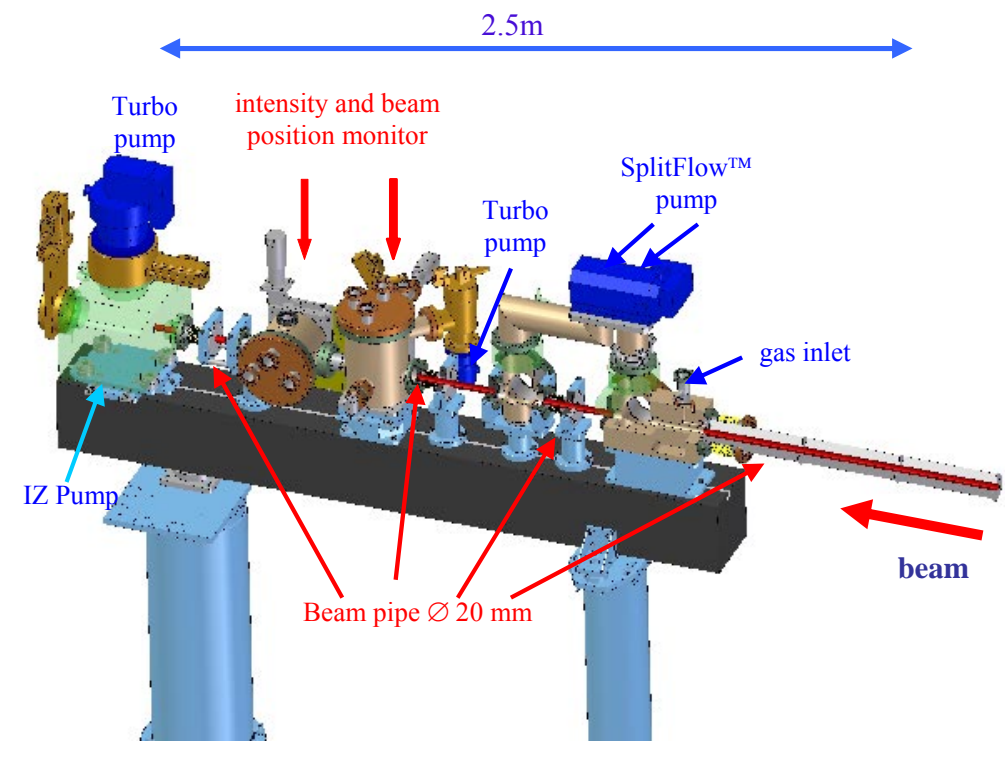

FIGURE 3. Differential pumping system at the entrance to the experimental hall. gas attenuator system, since this section is located in front of the photon beam distribution optics. Both sides of the windowless drift section, which is formed by a $\varnothing 100 \mathrm{~mm}$ beam pipe, are terminated by a differential pumping stage to preserve the beamline vacuum. Thus, the whole drift section of PETRAII can act as a gas cell. Figure 3 shows one differential pumping stage with integrated intensity and beam position monitor (GMD)[2]. The system is arranged in four pumping sections. Each of these sections is connected by conductance limiting pipes with inner diameters of $20 \mathrm{~mm}$. Since the lower limit of the inner diameter is defined by the clear aperture of the optical system, the lengths of the pipes are determined by the desired 


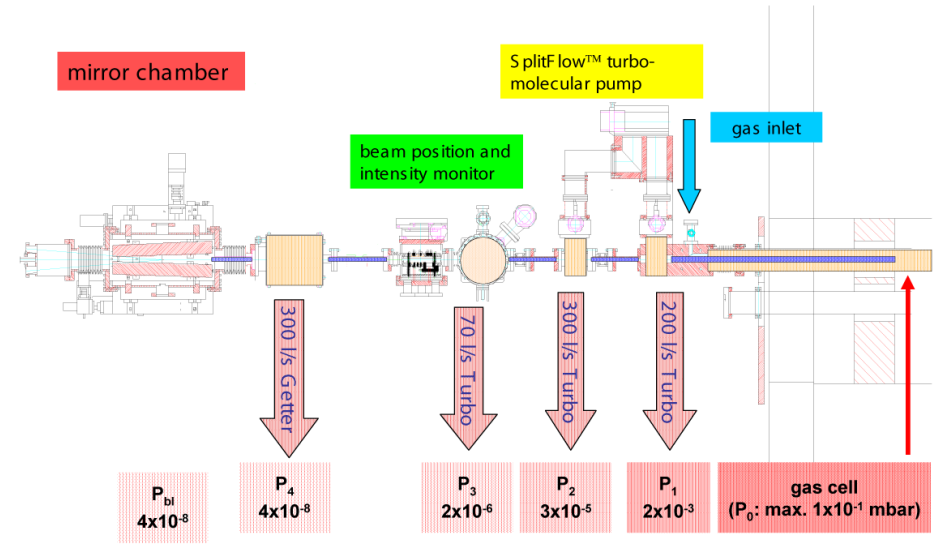

FIGURE 4. Calculated pressure distribution of the gas attenuator for $\mathrm{N}_{2}$ pressure gradient of each pumping section. We installed three pumping steps. One section, which is used for the GMD device, does not contribute to the differential pumping. The whole pumping system has to cover 8 orders of magnitude pressure gradient between beamline and gas cell. Therefore, each real pumping step has to reduce the pressure by a factor of $10^{2}$. In the molecular flow regime this factor is achieved by pumping speeds $>100 \cdot 1 / \mathrm{s}$ and $\sim 250 \mathrm{~mm}$ long $(20 \mathrm{~mm} \varnothing)$ pipes reducing the conductance to about $1 \cdot 1 / \mathrm{s}$. The first pumping step is operated in the transition range between $0.1 \mathrm{mbar}$ in the gas cell and $2 \cdot 10^{-3}$ mbar in the beamline. Conductance calculations [3] for $\mathrm{N}_{2}$ in the Knudsen range result in a length of $1.3 \mathrm{~m}$ to achieve a conductance of $1 \cdot 1 / \mathrm{s}$. A scheme of the calculated pressure distribution along the differential pumping unit is shown in Fig. 4. The arrows represent the applied pumping speeds and the underneath panels show the resulting pressures in each section. A pumping speed of $70 \mathrm{l} / \mathrm{s}$ at the GMD position is required to regulate the pressure in this diagnostic device. All the pipes have to be aligned with a tolerance of $<0.3 \mathrm{~mm}$ in order to avoid perturbations of the photon beam.

\section{TECHNICAL REALIZATION AND FIRST MEASUREMENTS}

\section{Pumping}

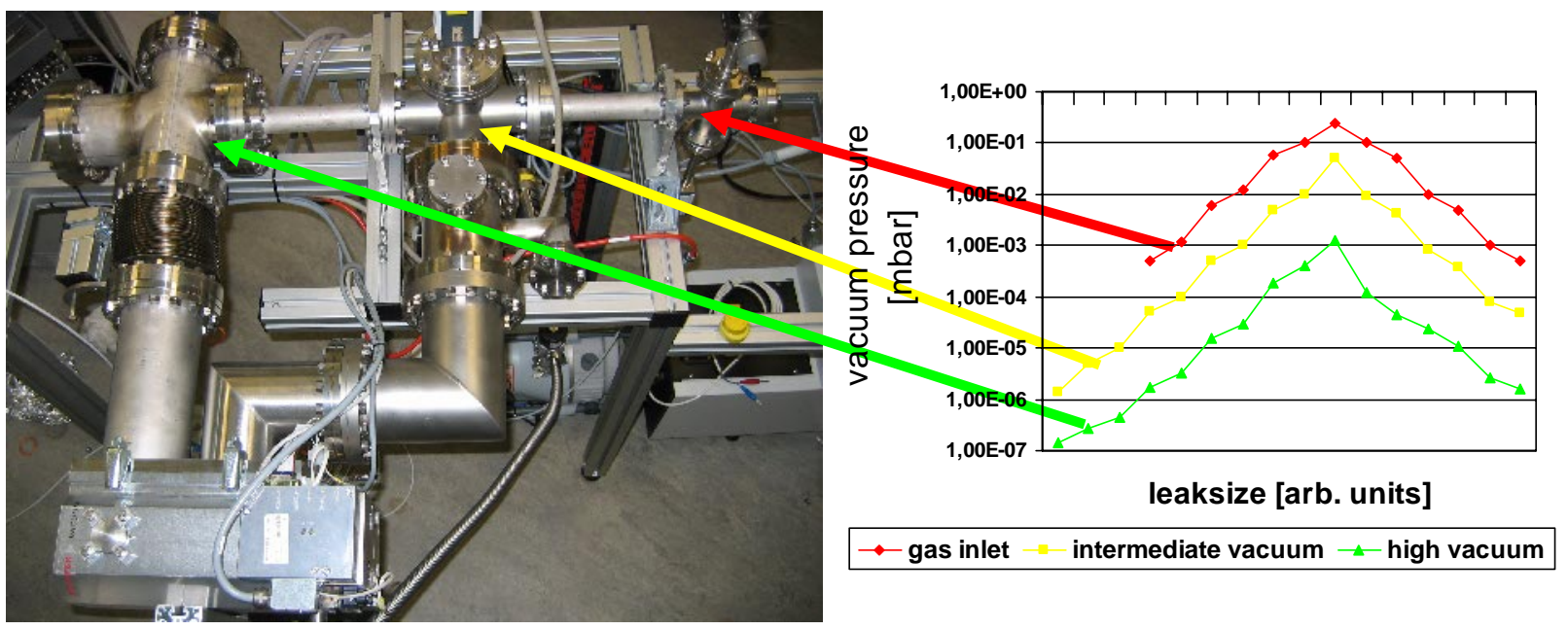

FIGURE 5. Set up to investigate pumping capabilities of the SlitFlow ${ }^{\mathrm{TM}}$ turbomolecular drag pump [4]. On the right are measurements of the inlet pressure (diamonds), the pressures on the intermediate (squares) and the high vacuum port (triangles).

At the high pressure side we have chosen a SplitFlow ${ }^{\mathrm{TM}}$ turbomolecular drag pump, which serves the two "high" pressure pumping ports. The capability of the SplitFlow ${ }^{\mathrm{TM}}$ pump has been tested in the setup shown in Fig. 5. Here, the pumping ports and the gas inlet are connected with a conductance of $\sim 10 \cdot 1 / \mathrm{s}$. The graph on the right depicts the results of the measurements. It shows that the intermediate port of the pump is able to handle a gas pressure of $0.1 \mathrm{mbar}$. 
The interface section to the beamlines is equipped with a 3001/s noble diode ion getter pump in inline configuration (the beam passes directly the pump body). Noble diode ion pumps are chosen, because of their high stability when pumping rare gases. This inline pump is supplied with an additive $\varnothing 150 \mathrm{~mm}$ flange to enable the installation of an additional turbomolecular pump (see Fig. 3), which will support and stabilize the pumping system in case of highest rare gas load.

\section{Alignment}

A crucial point is the alignment of the narrow and long pipes of the differential pumping set up. All components of the differential pumping system have been placed, aligned and fixed on granite girders (see Fig. 3) before setting in place. Here, $\mathrm{T}-$ slots on the granite girders allow precise alignment by tongue and groove joints in an easily reproducible way. The joints guarantee an exact positioning $(<0.3 \mathrm{~mm})$ of the installed components with respect to the beamline axis. The alignment of the girders on the other hand is realized by a special supporting structure. This procedure saves a lot of individual alignment work. Due to technical reasons the $1.3 \mathrm{~m}$ long pipe (Fig. 3) is supported on the gas inlet side only. To minimize the bending of the pipe a sophisticated supporting rip structure was designed. This enables a deflection free installation of the beampipe.

\section{First Results}

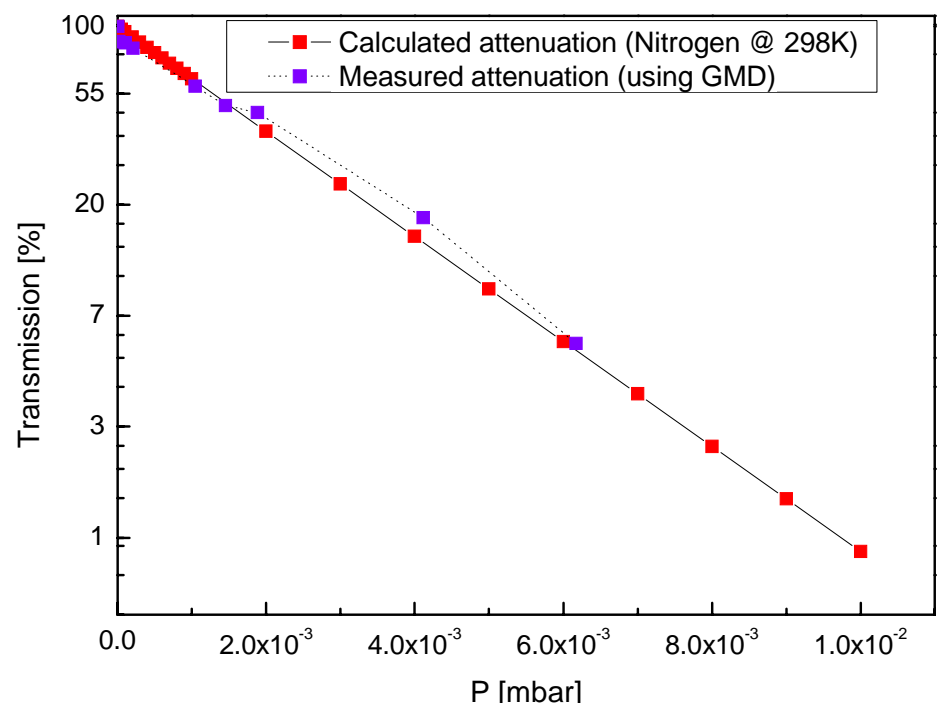

Figure 6 depicts the comparison between the calculated and measured transmission of the gas attenuator for a photon wavelength of $30 \mathrm{~nm}$. The transmission has been determined with help of the GMDs in front and behind the gas attenuator. The gas pressure was controlled by a gas dosing valve. The pressure readings and the transmission are plotted on double logarithmic scales. The plot shows a fairly good agreement between the calculated and the measured transmission. The detection efficiency of the second GMD set a lower limit of 5\% for the transmission measurement. The overall good agreement allows to extrapolate the expected transmission to higher gas cell pressures.

FIGURE 6. First transmission results with the gas attenuator

\section{REFERENCES}

1. V. Ayvazyan et al., First operation of a Free-Electron Laser generating GW power radiation at $32 \mathrm{~nm}$ wavelength, Eur. Phys. J. D 37, (2006), 297-303

2. A.A. Sorokin, S.V. Bobashev, J. Feldhaus, Ch. Gerth, A. Gottwald, U. Hahn, U. Kroth, M. Richter, L.A. Shmaenok, B. Steeg, K. Tiedtke and R. Treusch, Gas-Monitor Detector for Intense and Pulsed VUV/EUV Free-Electron Laser Radiation, Proceedings SRI2003, San Francisco, AIP Conf. Proc. 705 (2004), 557-560

3. M. Wutz, H. Adam, W. Walcher, and K. Jousten, Handbuch Vakuumtechnik, Vieweg Verlag, ISBN 3-528-54884-3, (2000)

4. SplitFlow ${ }^{\mathrm{TM}}$ turbomolecolar drag pump, TMH 261-250-010P, Pfeiffer Vacuum Techology AG, www.pfeiffer-vacuum.net 